\title{
Caudal Epidural Blood Patch FOR SPONTANEOUS INTRACRANIAL Hypotension in a Patient Receiving Dual Antiplatelet Therapy
}

\author{
George M. Jeha, BS ${ }^{1}$ \\ Jihad $G$. Jiha, MD² \\ Steven Zuckerman, $\mathrm{MD}^{3}$ \\ Alan D. Kaye, MD, $\mathrm{PhD}^{4}$
}

Background: Epidural blood patch (EBP) is routinely used to treat spontaneous intracranial hypotension $(\mathrm{SIH})$. In the case of a patient receiving dual antiplatelet therapy, a caudal EBP was utilized for treatment of his SIH-related chronic headache as the traditional lumbar interlaminar approach was not advisable due to bleeding risk. While there are case reports supporting the efficacy of caudal EPB for treating $\mathrm{SIH}$, none of the cases were performed in patients receiving antiplatelet therapy. In this case, we were uncertain whether antiplatelet agents would interfere with clot formation in the epidural space and preclude successful blood patch formation.

Case Report: Herein, we present a 50-year-old man diagnosed with SIH after presenting with a 2-month history of intractable, orthostatic, bifrontal headache. He had undergone coronary stenting 37 days prior and was receiving dual antiplatelet therapy with aspirin and clopidogrel. After the patient failed conservative measures, he elected to proceed with caudal EBP. This provided only 2 hours of symptom relief. One week later, another EBP was performed, this time with complete resolution of the patient's headache.

Conclusion: Our case demonstrates that the caudal approach to EBP may be a safe and effective alternative to interlaminar blood patch for treatment of spontaneous intracranial hypotension in patients at increased bleeding risk related to antiplatelet therapy.

Key words: Epidural blood patch, caudal blood patch, CSF leak, postural headache, SIH, spontaneous intracranial hypotension, anticoagulation

\footnotetext{
From: 'Louisiana State University School of Medicine, New Orleans, LA; 'Department of Anesthesiology, Louisiana State University School of Medicine, New Orleans, LA; ${ }^{3}$ Neuro Technology Institute, Baton Rouge, LA; ${ }^{4}$ Departments of Anesthesiology and Pharmacology, Toxicology, and Neurosciences, Louisiana State University School of Medicine, Shreveport, LA

Corresponding Author: Alan D. Kaye MD, PhD, Department of Anesthesiology and Pharmacology, Toxicology, and Neurosciences, Louisiana State University School of Medicine, 1501 Kings Hwy, Shreveport, LA 71103, Shreveport, LA, E-mail: akaye@lsuhsc.edu; alankaye44@hotmail.com Conflict of Interest: None Declared

Disclaimer: There was no external funding in the preparation of this manuscript. Conflict of interest: Each author certifies that he or she, or a member of his or her immediate family, has no commercial association (i.e., consultancies, stock ownership, equity interest, patent/licensing arrangements, etc.) that might pose a conflict of interest in connection with the submitted manuscript.
} 


\section{BACKGROUND}

Postural headaches related to reduced intracranial pressure were first described by Schaltenbrand in 1938 (1). Since then, spontaneous intracranial hypotension (SIH) has grown to become recognized as an important potential cause of severe persistent headache (2-4). The mechanism of chronic headache in patients with $\mathrm{SIH}$ is thought to be related to cerebrospinal fluid (CSF) hypovolemia secondary to spontaneous CSF leakage, which leads to downward displacement of the brain and subsequent traction on supporting pain-sensitive brain structures, particularly the meninges and bridging veins $(2,5,6)$. A second proposed mechanism involves dilation of intracranial pain-sensitive venous structures in response to intracranial hypotension (5). The incidence of $\mathrm{SIH}$ is 5 in 100,000 and peaks at 40 to 50 years (3-5). Numerous terms such as "low CSF volume headache," "CSF hypovolemia," "CSF volume depletion," and "spontaneous CSF leak" have been used to describe this syndrome and these terms may be used interchangeably (7). The diagnostic hallmark of SIH, although not clinically present in all cases, is the triad of orthostatic headache, diffuse patchy meningeal enhancement on imaging, and low CSF opening pressure (6). Orthostatic headache is the most commonly reported clinical manifestation of $\mathrm{SIH}$, although additional symptoms such as neck pain or stiffness, nausea, and vomiting may occur in up to half of patients $(5,7)$. Rarely, coma and even death have been described $(8,9)$.

The headache of $\mathrm{SIH}$, which may be diffuse in nature or may localize to the occipital, suboccipital, temporal, or frontal areas, characteristically worsens within 15 minutes of sitting or standing and improves within minutes after assuming the recumbent position (5-7). When the headache fails to resolve spontaneously or with conservative measures (including bed rest, caffeinated products, hydration, or medications), epidural blood patch (EBP) is the treatment of choice. The procedure involves placing a needle in the epidural space through an interlaminar approach and slowly (over a course of 30 to 60 seconds) injecting approximately $20 \mathrm{~mL}$ of sterile autologous blood (10). There are 2 plausible explanations for the therapeutic effect of EBP: an early effect may simply be related to volume replacement, while a delayed effect may involve sealing of the CSF leak by the blood patch (6). Many cases require placement of a second or multiple EBPs (4-6). While the lumbar interlaminar approach is most commonly utilized, there are certain conditions that preclude this approach.
Examples of circumstances in which interlaminar EBP may be impractical or inadvisable include infection at the injection site, the presence of surgical wounds or scarring, or the use of anticoagulation agents (10-14). We report a case of successful administration of EBP via a caudal approach for treatment of SIH-related chronic headache in a patient receiving dual antiplatelet therapy with aspirin and clopidogrel. Administration through the sacral hiatus minimized the risk of bleeding compared with the traditional lumbar interlaminar approach. Despite the presence of antiplatelet agents, a caudal EBP was successful in resolving this patient's chronic headache.

\section{CASE REPORT}

A 50-year-old male boat pilot with a past medical history significant for type II diabetes mellitus and coronary artery disease was seen by his neurologist for a 2-month history of severe, daily headaches. The patient had no neurological deficits, mental status changes, or vision changes. He had no history of migraines, tension headaches, or cluster headaches. Thirty-seven days prior to evaluation, the patient had undergone coronary angioplasty with stent placement and was receiving dual antiplatelet therapy with clopidogrel (75 mg daily) and aspirin (81 mg daily). The headache was bifrontal in location, exacerbated with sitting up or standing, and alleviated with lying down. Upon further questioning, the patient recalled an incident during which he sustained a fall onto his buttocks, and he states that his headaches began shortly afterwards. Additionally, he endorsed intermittent nasal discharge of clear, watery fluid. Computed tomography scan of the head with contrast medium showed no evidence of active CSF leak. Nonetheless, given the nature of this patient's headaches, the clinical suspicion for low intracranial pressure remained high, and cisternography was considered to evaluate for CSF leak. After speaking with the patient's cardiologist, however, it was established that the patient's dual antiplatelet therapy should not be interrupted, and this limited our ability to perform diagnostic interventional procedures.

Initially, conservative treatment was attempted for treating the patient's headaches. Rest, hydration, caffeinated products, and analgesics were ineffective in controlling his symptoms. Twelve weeks later, he was still experiencing significant pain despite the use of hydrocodone-acetaminophen tablets more than twice per day. At this time, the idea of attempting EBP 
via a caudal approach despite continuation of dual antiplatelet therapy was discussed with the patient. It was explained that the effectiveness of EBP in patients receiving dual antiplatelet therapy was not well established. The patient was informed of the risks and benefits of the elected procedure including but not limited to: increased headache, no pain relief, bleeding, infection, nerve injury, dural puncture, potential paralysis, loss of use of one or more extremities, stiff neck/back, medication reaction, muscle spasms, and difficulty sleeping. The patient understood, was given opportunity for question/answer dialogue, and elected to proceed with caudal EBP (Fig. 1).

Initial caudal EBP provided only 2 hours of symptom improvement. One week later, a second caudal EBP was performed. In the postanesthesia care unit, the patient reported significant relief of his headache. Pain was reduced from 7 out of 10 on the Visual Analog Scale before the procedure to 0 out of 10 after the procedure in both the sitting and standing positions. One week later, the patient reported continued $100 \%$ relief of his headache. He was able to perform his activities of daily living with no complications except for some occasional soreness in the lower back when lying flat.
Eight weeks after the second EBP, the patient continued to be without headaches and had returned to his job as a boat pilot on the Mississippi river after missing work for almost 3 months.

\section{DISCUSSION}

The patient described in this case posed a therapeutic conundrum for his treatment team. On one hand, the patient was experiencing daily, debilitating headache for which he had failed conservative therapy and for which EBP seemed the most promising intervention. On the other hand, performing this procedure while the patient was undergoing treatment with dual antiplatelet agents raised concerns about increased risk for bleeding and the possible devastating complication of epidural hematoma. There was also the possibility that the presence of antiplatelet agents would inhibit platelet aggregation and clot formation, in which case we would be exposing the patient to the risks of a procedure that may ultimately be rendered therapeutically futile. In some circumstances, temporary cessation of antithrombotics or anticoagulants is recommended prior to undergoing interventional procedures $(15,16)$. However, we were hesitant to make alterations to the

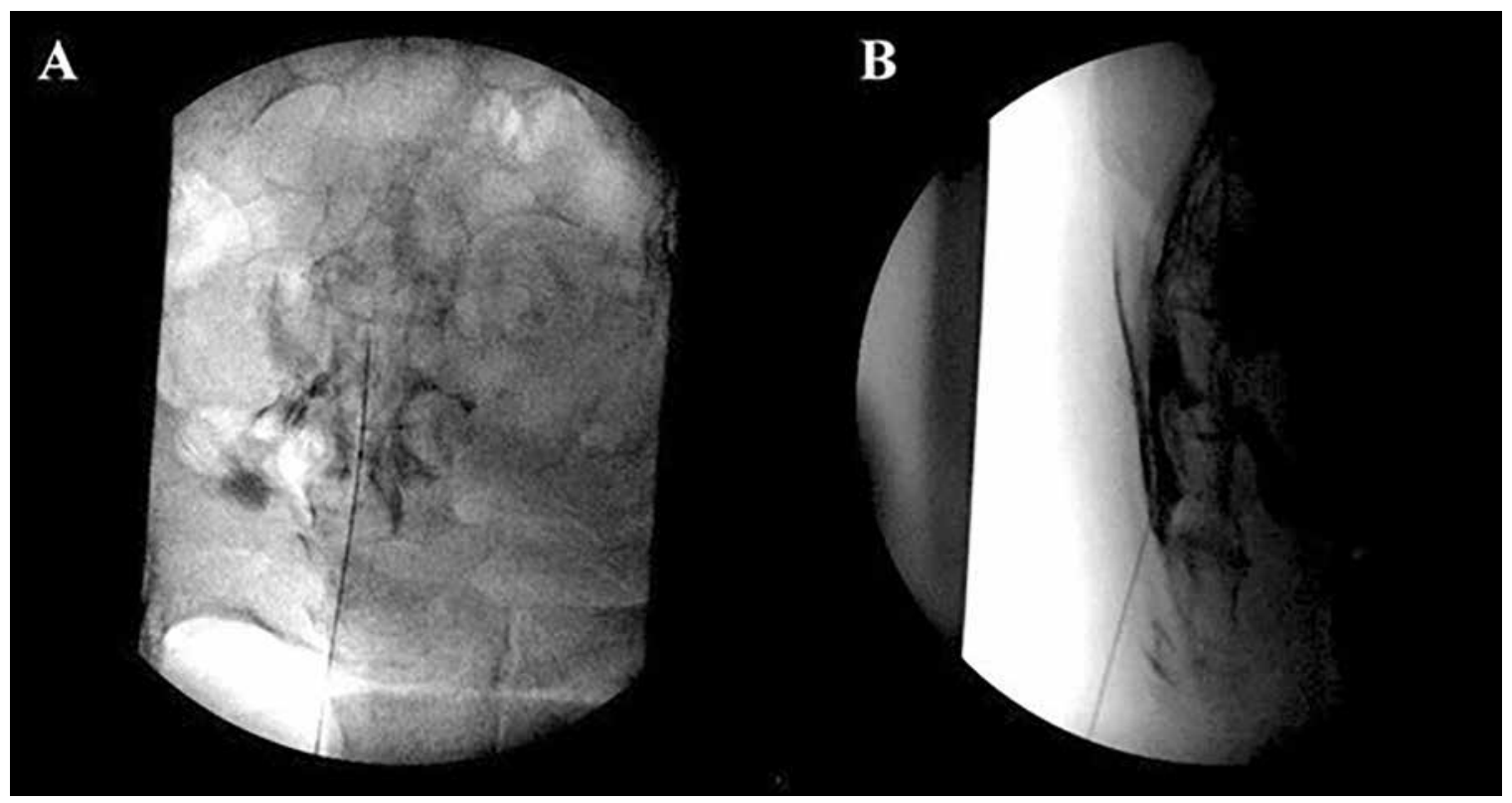

Fig. 1. Caudal fluoroscopy of the patient using iohexol $300 \mathrm{mg} / \mathrm{mL}$ contrast medium confirming appropriate needle placement prior to the injection of blood into the epidural space. Anterior-posterior view (Fig. 1A) and lateral view (Fig. 1B). 
patient's antiplatelet regimen since he had undergone placement of a coronary stent only 37 days prior. While the highest rates of coronary stent rethrombosis occur in the first 30 days after stent placement, the patient was still well within the 12-month period during which dual antiplatelet therapy is recommended $(17,18)$.

Because a caudal approach for epidural steroid injections poses less bleeding risk than lumbar interlaminar injections, we felt that the caudal approach for EBP may provide therapeutic response while minimizing bleeding risk. Although we were encouraged by several reports in the literature of successful treatment of $\mathrm{SIH}$ via caudal EBP, none of these cases were attempted in anticoagulated patients. Thus, it was uncertain whether the blood patch would prove successful in an anticoagulated patient. Our patient had exhausted alternative treatment options and was still experiencing daily, debilitating headaches which were negatively impacting normal daily function. After he was made aware of the risks, benefits, and possible complications and outcomes of caudal EBP placement, including the possibility that the procedure may be unsuccessful in resolving his headaches, the patient elected to undergo this procedure.

In a 2019 update, the American Society of Interventional Pain Physicians provided guidelines for the use of antithrombotics and anticoagulants in patients undergoing interventional techniques (19). While these guidelines do not address EBP specifically, they classify lumbar interlaminar epidural injections as intermediaterisk procedures based on the potential risk for bleeding. Meanwhile, caudal epidural injections are classified as low-risk procedures. For this reason, caudal EBP was chosen for the current patient in order to minimize the risk of bleeding.

The first use of caudal EBP for treating SIH was reported in 1998 by Gerancher et al (20) in a 28-yearold woman with scoliosis who developed postdural puncture headache following 4 unsuccessful attempts at delivering epidural labor analgesia. Given the difficulties with identification of the epidural space, the patient underwent EBP via a caudal approach with catheter guidance. Caudal EBP with catheter guidance has since been reported in a pediatric patient with acute lymphocytic leukemia who developed subarachnoid-cutaneous fistula and continuous CSF leak secondary to repeated lumbar punctures for intrathecal administration of chemotherapy, and also in a 2019 case report involving a postpartum woman with previous spinal surgery who developed postdural puncture headache $(21,22)$. In another report, the authors mixed autologous blood with contrast media prior to injection in an attempt to identify the precise location of blood patch formation within the epidural space (11). Although these cases were not performed in anticoagulated patients, they spoke to the feasibility of caudal EBP placement and inspired us to pursue this approach in our patient. The patient experienced transient relief after the initial blood patch placement and long-term relief after the second blood patch placement, with no symptoms after 8 weeks of follow-up. At no point was there evidence of any bleeding event even though the patient continued his daily antiplatelet regimen as prescribed.

Aspirin, a direct inhibitor of cyclooxygenase (COX), irreversibly inhibits the enzyme via acetylation and disrupts production of thromboxane A2, a substance which triggers platelet aggregation, vasospasm, and serotonin release from platelets. Subsequently, the ratio of prostacyclin to thromboxane $A 2$ is increased, resulting in reduced thrombus formation and clotting (23). Clopidogrel belongs to a class of medications termed thienopyridine inhibitors. These exert their antiplatelet effect by binding to $\mathrm{P} 2 \mathrm{Y} 12$ receptors, which irreversibly alters and significantly inhibits platelet aggregation (24). There are numerous other antithrombotics and anticoagulants available which affect various stages in the platelet aggregation pathway and the coagulation cascade. While aspirin and clopidogrel did not preclude successful blood patch formation in our described patient, this does not speak to the feasibility, efficacy, or safety of the procedure in patient receiving other agents.

\section{LIMITATIONS}

In the current case, diagnostic myelography, cisternography, or lumbar puncture were not performed due to the patient's antiplatelet regimen. This limited our ability to meet the diagnostic criteria to definitively establish a diagnosis of SIH. However, given the clinical characteristics of the patient's headache and response to EBP, the authors believe that the current case is most consistent with $\mathrm{SIH}$.

\section{CONCLUSION}

Despite our concern that this patient's concomitant dual antiplatelet regimen would interfere with blood patch formation, we were able to achieve clinical resolution of this patient's chronic headache after performing 
2 caudal EBPs with no adverse bleeding event. Our case demonstrates that caudal EBP may be a safe and effective alternative to interlaminar blood patch for treatment of spontaneous intracranial hypotension in patients receiving dual antiplatelet therapy.

\section{REFERENCES}

1. Schaltenbrand VG. Neuere anschauugen zur pathophysiologie der liquorzirkulation. Zentralbl Neurochir 1938; 3:290-299.

2. Annabi E, Bowden K, Wuollet A, et al. Transforaminal blood patch for the treatment of chronic headache from intracranial hypotension: A case report and review. Anesthesiol Res Pract 2012; 2012:923904.

3. Amrhein TJ, Kranz PG. Spontaneous intracranial hypotension: Imaging in diagnosis and treatment. Radiol Clin North Am 2019; 57:439-451.

4. Schievink WI, Maya MM, Moser F, Tourje J, Torbati S. Frequency of spontaneous intracranial hypotension in the emergency department. J Headache Pain 2007; 8:325-328.

5. Schievink WI. Spontaneous spinal cerebrospinal fluid leaks and intracranial hypotension. JAMA 2006; 295:2286-2296.

6. Mokri B. Spontaneous CSF leaks: Low CSF volume syndromes. Neurol Clin 2014; 32:397-422.

7. Kwak KH. Postdural puncture headache. Korean J Anesthesiol 2017; 70:136-143.

8. Sayer FT, Bodelsson M, Larsson EM, Romner B. Spontaneous intracranial hypotension resulting in coma: Case report. Neurosurgery 2006; 59:E204.

9. Francia A, Parisi P, Vitale AM, Esposito V. Life-threatening intracranial hypotension after diagnostic lumbar puncture. Neurol Sci 2001; 22:385-389

10. Ioscovich A, Giladi Y, Fuica RL, et al. Anesthetic approach to postdural puncture headache in the peripartum period: An Israeli national survey. Acta Anaesthesiol Scand 2018; 62:1460-1465.

11. Chen IW, Lin CM. Fluoroscopy-guided caudal epidural blood patch for relieving post-dural puncture headache after lumbar spine surgery. Egypt J Anaesth 2017; 33:133-135.

12. Fujiwara A, Watanabe K, Hashizume K, et al. Transforaminal epidural blood patch for intractable spontaneous cerebrospinal fluid leak: A case report. JA Clin Rep 2017; 3:3-7.

13. Weil L, Gracer RI, Frauwirth N. Transforaminal epidural blood patch. Pain Physician 2007; 10:579-582.
14. Park $\mathrm{CH}$, Lee $\mathrm{SH}$. Lumbar transforaminal epidural blood patch. Pain Med 2014; 14:945-947.

15. Novitch M, Hyatali FS, Jeha G, et al. Regional techniques for head and neck surgical procedures. Best Pract Res Clin Anaesthesiol 2019; 33:377-386.

16. Patel RB, Tassiopoulos AK. Anticoagulants and surgery: So many agents, so many taking them. Adv Surg 2019; 53:235-251.

17. Ludman PF. Percutaneous coronary intervention. Medicine 2018; 46:547-554.

18. Levine GN, Bates ER, Bittl JA, et al. Clinical statements and guidelines. Circulation 2016; 134:123-155.

19. Kaye AD, Manchikanti L, Novitch MB, et al. Responsible, safe, and effective use of antithrombotics and anticoagulants in patients undergoing interventional techniques: American Society of Interventional Pain Physicians (ASIPP) Guidelines. Pain Physician 2019; 22:S75-S128.

20. Gerancher JC, D'Angelo R, Carpenter R. Caudal epidural blood patch for the treatment of postdural puncture headache. Anesth Analg 1998; 87:394-395.

21. Kowbel MAM, Comfort VK. Caudal epidural blood patch for the treatment of a paediatric subarachnoid-cutaneous fistula. Can $J$ Anaesth 1995; 42:625-627.

22. Orza FM, Averbakh E, Todd MM. Postdural puncture headache in a postpartum female with previous spinal surgery-successful treatment with caudal epidural blood patch: A case report. A A Pract 2019; 12:57-58.

23. Bates SM, Greer IA, Middeldorp S, Veenstra DL, Prabulos AM, Vandvik PO. VTE, thrombophilia, antithrombotic therapy, and pregnancy. Chest 2012; 141:e691S-e736S.

24. Rao GHR, Divani A. Past, present, and future of anti-platelet therapy. J Vasc Interv Neurol 2008; 1:57-60. 
Pain Medicine Case Reports 